\title{
CINEOLE, THYMOL AND CAMPHOR NASAL CHALLENGES AND THEIR EFFECT ON NASAL SYMPTOMS AND COUGH IN AN ANIMAL MODEL
}

\author{
Gavliakova S, Dolak T, Licha H, Krizova S, Plevkova J.
}

Department of Pathophysiology, Jessenius Faculty of Medicine, Comenius University, Martin, Slovak Republic

\section{A b s t r a c t}

Inhalation of aromatic vapours suppressed coughing induced by citric acid (CA) in naive animals. No data are available about their effects in an animal model with primarily up-regulated cough reflex. New data indicate that aromatic vapours suppress cough via effect on nasal sensory nerves.

The aim of our study was to ascertain the efficacy of nasal application of 1,8-cineole, thymol and camphor on nasal symptoms and CA induced cough in validated model of up-regulated cough reflex. Guinea pigs ( $\mathrm{n}=13$ ) were sensitized by intraperitoneal administration of ovalbumin (OVA) and sensitization was confirmed 21 days later by skin tests. Sensitized animals were repeatedly challenged with nasal OVA to induce rhinitis, and further experiments (cough challenges) were performed during the early phase of allergic inflammation.

Cough was induced by CA in plethysmograph for 10 minutes after nasal pre-treatment with aromatic substances $\left(10^{-3} \mathrm{M}\right)$ in rhinitis model. Cough was recognized from record of sudden airflow changes interrupting breathing pattern and cough sound. Final count of coughs was established by blind analysis using SonicVisualiser Software. Dose responses curves, total cough count and cough latency were analyzed.

Repeated intranasal challenge with OVA induces progressively worsening symptoms, and cough induced by CA during acute phase of allergic rhinitis was enhanced. Nasal pre-treatment with 1,8-cineole, thymol and camphor did not prevent onset of nasal symptoms, and the magnitude of symptoms was comparable to those without pretreatment. Camphor had the most potent antitussive effects (number of coughs $25 \pm 3$ vs. $7 \pm 2$, p<0.05) followed by thymol (number of coughs $25 \pm 3$ vs. $14 \pm 2$, p $<0.05$ ). The data for nasal 1,8 -cineole challenge did not reach statistical significance. Cough latency followed this trend.

Although the magnitude of nasal symptoms is not influenced, the effect on cough is in case of camphor and thymol significant. Our data showed that nasal application of aromatic substances suppress citric acid induced cough in animals with up-regulated cough reflex.

Key words: cough - upper airways - cineole - camphor - thymol - animal models

\section{INTRODUCTION}

Interesting papers documenting the effects of herbal extracts on upper airway diseases including cough were published, sharing evidence about tolerability and efficacy of these extracts [1, 2, 3, 4, 5]. Thymol, camphor and 1,8-cineole are known as aromatic compounds, which have been widely used in the symptomatic treatment of upper respiratory tract diseases and cough despite little objective evidence as to their benefit. The evidence of antitussive effect of camphor and cineole in a vapour form was obtained in naive guinea pigs [6] and antitussive effect of thymol nasal challenge was documented in human healthy volunteers [7].

It is suggested that aromatic substances can suppress cough via their action on sensory nerves in the upper airways. This evidence was obtained for menthol and the data are supported by the sc-rt-PCR studies detecting high expression of TRPM8 channels on trigeminal sensory afferents [8]. Based on the data from human healthy volunteers, aromatic substances influence also the urge-to-cough [7,9]. This is circumstantial evidence that effect of aromatic substances on cough is complex, involving supramedullar mechanisms.

Thymol, 1,8-cineole and camphor exert characteristic olfactory sensation and when applied to the skin or mucosa, they have anti-irritating effect, which is accompanied by

A d d r e s f o r c o r r e s p o n d e n c e:

Assoc. Prof. Jana Plevkova,PhD. Department of Pathophysiology, Jessenius Faculty of Medicine, Comenius University, Sklabinska Str. 26, 03601 Martin, Slovak Republic; e-mail: jplevkova@gmail.com 
cooling sensation. The molecular background for this effect is the TRPM8 ion channel, which is activated by these natural ligands, and also by temperatures $\sim 25^{\circ} \mathrm{C}[10,11]$. Thermosensors are expressed in a subset of small diameter sensory neurons, which constitute a functionally distinct population [12]. Thymol activates TRPV3 channel which is expressed on skin, tongue, brain and afferent somatosensory neurons [13]. Camphor is also TRPV3 agonist, and same time it strongly desensitizes TRPV1 ion channel [14]. Activation of TRPV1 on vagus nerves directly triggers coughing, or up-regulates it, when nasal TRPV $1^{+}$ neurons are stimulated [15, 16].

It is well known that cough undergoes up and down regulation by the mechanisms affecting peripheral and/or central neuronal pathways involved in the neurogenesis of cough [17]. It has been documented already that stimulation of nasal afferents via TRPV1 receptor enhances the cough response [15] and stimulation of TRPM8 channels down - regulates it in healthy volunteers and naive animals $[8,9]$. No data are available about the effect of aromatic substances on cough, which is primarily up-regulated. This study was aimed to ascertain the effect of 1,8-cineole, thymol, and camphor on nasal symptoms and citric acid induced cough in animals with allergic rhinitis - a model validated by our colleagues [18].

\section{METHODS}

\section{General notes}

This study was conducted on male Dunking Hartley guinea pigs, obtained from an accredited breeding facility (L. Sobota, Městec Králové, Czech Republic). The animals were housed in an approved animal holding facility maintained at a controlled room temperature of 21$22^{\circ} \mathrm{C}$, with humidity $45 \pm 10 \%$, ventilation, a 12 -h light-dark cycle and had free access to water and standard animal food.

Animal care was provided and the experiments were conducted in agreement with the Animal Welfare Guidelines of the Comenius University and statutes and rules of the Slovak Republic legislation. The current study was approved by decision No: 2999/07-221.

Animals were adapted twice to laboratory conditions in order to significantly reduce future stress. They stayed in a plethysmographic box to familiarize themselves with the environment and the persons responsible for the experimental manipulations. In the plethysmographic box, they were exposed to aerosol of buffered saline for 2 minutes, which corresponded to the future experimental procedure.

\section{Model of airway hyperresponsiveness and sensitization}

Guinea pigs $(n=13)$ were sensitized with intra peritoneal injection of ovalbumin $(10 \mu g$, Sigma) administered with aluminium hydroxide in $1 \mathrm{ml}$ of saline [19]. Successful sensitization was confirmed after 21 days by OVA skin prick test and oedema, redness/flare and later infiltration were taken as evidence of successful sensitization. Allergic rhinitis was induced by intranasal instillation of the antigen - ovalbumin ( $15 \mu 1,0.5 \%$ OVA) and the symptoms and signs appear almost immediately after the exposure. Magnitude of the symptoms is individual, however, the duration of an acute episode of nasal symptoms lasted up to one hour in all animals with maximal magnitude after 15 min from the allergen exposure. This model of allergic rhinitis was developed based on the Underwood's study and modified and validated in our laboratory [18, 19]. The most important advantage of this model is up-regulation of cough, which allows to study the effects of defined aromatic substances.

Intranasal OVA challenge leads to nearly immediate response - since it induces nasal irritation, sneezing, nasal discharge and audible acoustic phenomena - crackles. The symptoms intensity was evaluated by trained persons used a nasal symptom score which was developed and validated by Brozmanova [18] matching symptoms intensity to numeric values giving maximum 6 and minimum 0 points of numeric scale as follows: 
Nasal discharge: no discharge 0, mild/moderate 1, discharge falls in drops from nose 2 Eye/conjunctiva: no changes 0 , hazy eyes 1 , visible lacrimation 2

Nasal phenomenon: no crackles 0 , audible crackles 1, crackles audible from a distance 2

\section{Nasal pre-treatment and protocol of the study}

Citric acid - induced cough was assessed five times during the study, always in a week intervals to avoid tachyphylaxis of the airway cough mediating afferents and maintain good reproducibility of the cough response. The cough challenges were randomized, however, the final expression of the data is in order

Saline nasal challenge (negative control) - sensitized animals, but no rhinitis

OVA nasal challenge (positive control) - sensitized animals with rhinitis

OVA $+1,8$-cineole - animals with rhinitis pre-treated with 1,8-cineole

OVA + thymol - animals with rhinitis pre-treated with thymol

OVA + camphor- animals with rhinitis pre-treated with camphor

This design was selected to ascertain the effects of selected aromatic substances on citric acid induced cough. Aromatic compounds were administered to the both nostrils $(0.015 \mathrm{ml}$, $10^{-3} \mathrm{M} 2 \mathrm{~min}$ prior to the OVA challenge and the coughing was induced after 15 minutes after the onset of nasal symptoms or in due time interval in control challenge. Concentrations were selected based on our previous studies with menthol and other aromatic substances.

\section{Citric acid - induced cough in conscious guinea pig model}

The awake animals $(n=13)$ were individually placed in the plethysmograph (type 855, Hugo Sachs Electronic, Germany) which consists of a head chamber and a body chamber. The opening between the head chamber and body chamber was equipped with a plastic collar lining around the animal's neck to prevent communication between the chambers. The appropriate collar size was chosen for each animal to prevent neck compression. The head chamber was connected to a nebuliser (Pari Provokation Test I, Menzel, Germany, manufacturer's specification: output $51 . \mathrm{min}^{-1}$, particle mass median aerodynamic diameter $\left.1.2 \mu \mathrm{m}\right)$. A suction device adjusted to the same input (5 $\left.1 . \mathrm{min}^{-1}\right)$ was connected to the head chamber to maintain constant airflow through the chamber during the aerosol administration.

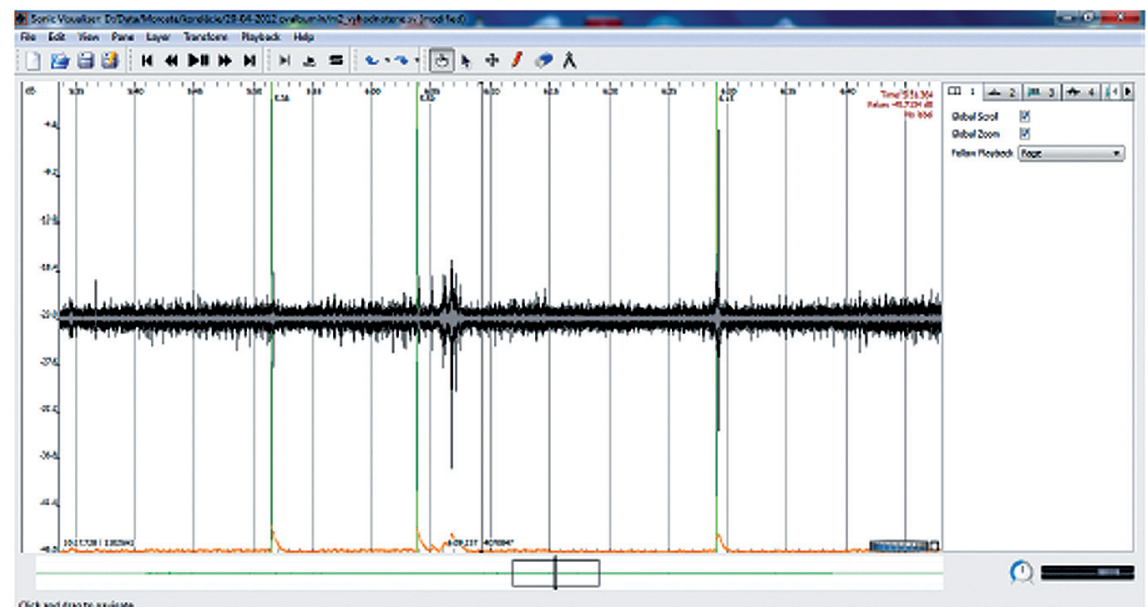

Fig. 1 The screen of the software Sonic Visualiser, which is helpful tool in assessment of the cough sound, as the second characteristic phenomenon of coughing. Cough sound is produced by the resonances of the airway structures, mainly structures of the supralaryngeal vocal tract. It also has a typical power spectrum, but Fast Fourrier Transformation provided specificity at the level of trained observer - person experienced in cough sound analysis. Sonic Visualiser is used together with the data for the airflow obtained by ACQ Knowledge. Matching the airflow and sound optimizes the results for final count of coughs. 
Respiratory changes, in particular airflow, were measured using a pneumotachograph (Godart, Germany) with a Fleisch head connected to the head chamber. The data were recorded with the acquisition system ACQ Knowledge (Biopack, Santa Barbara, CA, USA). Respiratory sounds, including sounds during coughing and sneezing, were recorded by a microphone placed in the roof of the head chamber and connected to a preamplifier and MP3 recorder. The pneumotachograph and microphone output were simultaneously recorded for off-line analysis.

The cough challenge was performed using an inhalation of $0.4 \mathrm{M}$ citric acid for $10 \mathrm{~min}$. Cough was defined as expiratory airflow interrupting the basic respiratory pattern accompanied by a coughing sound. Coughs were analyzed using cough-related sounds (Sonic Visualiser Software) (Fig. 1) and airflow, by two trained persons, both were blind to the carried out procedures. Their results were compared and (if no statistically significant differences occurred) averaged. The animals were challenged by citric acid (CA) several times, always with a one week interval between the challenges. The cough challenges began after the onset of nasal symptoms in animals with rhinitis or in a due time interval in pre-treated animals or control challenge.

\section{Reagents}

Citric acid was purchased from Fisher (Slovak Republic) and freshly diluted to $0.4 \mathrm{M}$ in distilled water at the day of use. OVA, 1,8-cineole, thymol and camphor were purchased from Sigma-Aldrich (Slovak Republic). 1,8-cineole, thymol and camphor were dissolved to the stock solutions and further diluted to $10^{-3} \mathrm{M}$ at the day of use. Aromatic substances $\left(10^{-3} \mathrm{M} ; 0.015 \mathrm{ml}\right.$ of each) were instilled locally to the nostrils using thin flexible catheter.

\section{Statistical analysis}

For count of coughs and cough latency data non- parametric not- paired tests and multiple comparison ANOVA tests were used as appropriate. Data for final count of coughs and cough latency are expressed as median \pm interquartile range or mean \pm standard error of mean, respectively. $\mathrm{P}<0.05$ was considered as statistically significant.

\section{RESULTS}

\section{Effects of aromatic substances on upper airway symptoms}

Nasal administration of ovalbumin induced reproducible nasal symptoms such are irritation, sneezing, nasal discharge, nasal crackles and ocular symptoms starting with hazy eyes, leading to fully developed conjunctivitis. All mentioned symptoms were carefully observed and recorded by trained staff during fully developed rhinitis with no treatment, and then in animals with rhinitis, but pre-treated with selected aromatic substances. Nasal pre-treatment with camphor, thymol and 1,8-cineole did not minimize the nasal symptoms, and the symptom score was similar after all observations. No tendency to improvement was observed after all pre-treatments. Data not shown.

\section{Effect of aromatic substances on cough latency and total cough response}

Cough latency was measured from the beginning of the citric acid challenge until the first cough appeared. The data are indicative for the strong anti-irritating effect of the aromatic substances. Cough latency after intranasal administration of saline was $2.5 \pm 0.3 \mathrm{~min}$, and it declined to $1.5 \pm 0.5$ min after nasal allergen challenge followed by rhinitis documenting predisposition for the earlier cough appearance after the exposure to the tussive aerosol of citric acid. The pre-treatment with 1,8-cineole, thymol and camphor prolonged the period the animals remained free off coughing after the exposure to citric acid to $2 \pm 0.4 \mathrm{vs}$. $3.3 \pm 0.3$ vs. $2.8 \pm 0.3, \mathrm{p}<0.05$ for thymol and camphor, data for 1,8-cineole did not reach statistical significance (Fig. 2). 


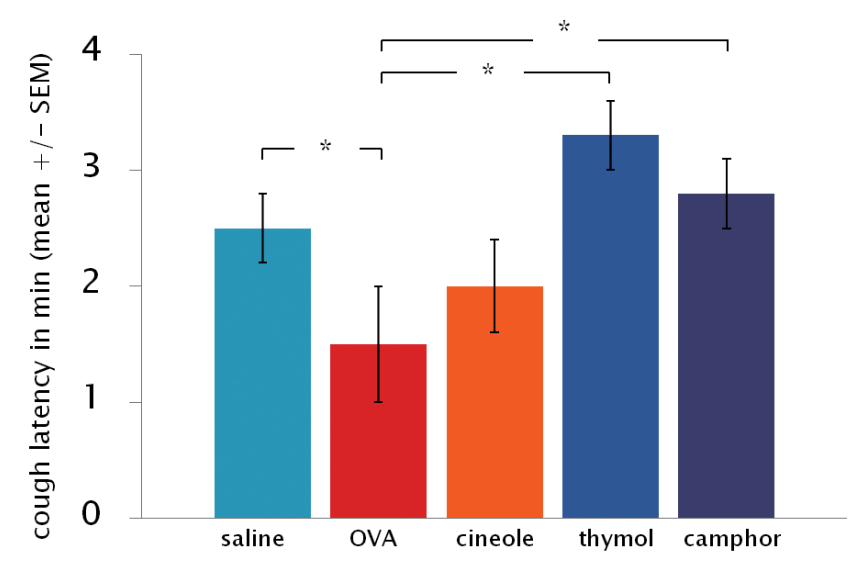

Fig. 2 The data or the cough latency measured from the start of the exposure to citric acid. Cough latency after intranasal administration of saline was $2.5 \pm 0.3 \mathrm{~min}$, and it declined to $1.5 \pm 0.5 \mathrm{~min}$ after nasal allergen challenge and rhinitis documenting the predisposition for the earlier cough appearance after the exposure to the tussive aerosol in animals with upper airway pathology. The pre-treatment with 1,8-cineole, thymol and camphor prolonged the period the animals remained free off coughing after the exposure to citric acid to $2 \pm 0.4$ vs $3.3 \pm 0.3$ vs $2.8 \pm 0.3, p<0.05$ for thymol and camphor, data for 1,8 -cineole did not reach the level of statistical significance. * $\mathrm{p}<0.05$, SEM - standard error of mean, OVA - ovalbumin

Total count of coughs measured during 10 min exposure to citric acid followed this pattern of changes. Number of coughs obtained during control condition after nasal administration of saline was $10 \pm 2$ coughs and this number increased to the $25 \pm 3$ coughs after nasal allergen challenge. Significant up-regulation of cough was expected to appear and it demon-

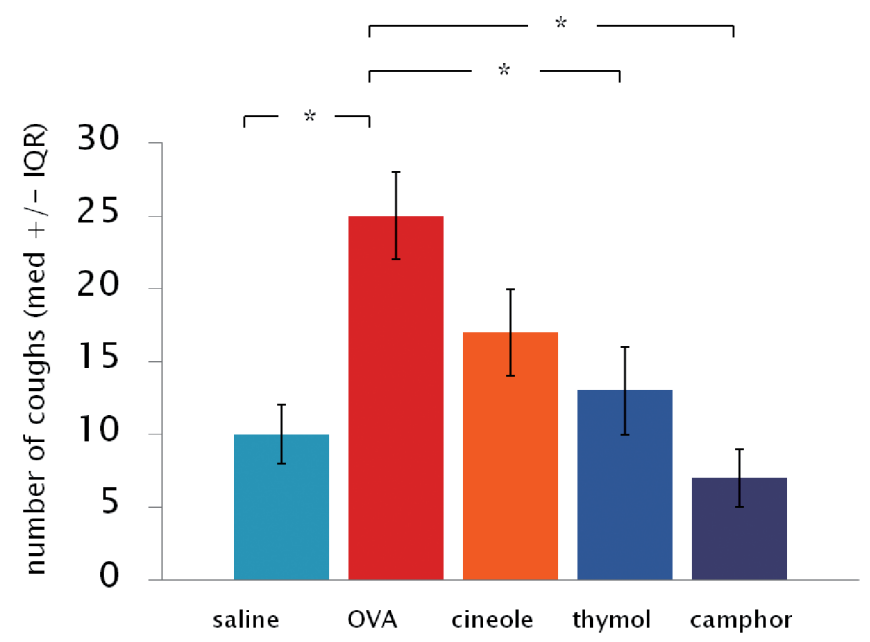

Fig. 3 The total count of the cough efforts obtained in a provocation with $0.4 \mathrm{M}$ citric acid in animals with intranasal saline, OVA, and animals with rhinitis, but pretreated with aromatic substances. See legend. Number of coughs obtained during control condition after nasal administration of saline was $10 \pm 2$ coughs and this number arose to the $25 \pm 3$ coughs after nasal allergen challenge. Significant up-regulation of cough was expected and it demonstrates the effect of pathological process located in the upper airways on coughing. Observed reduction of coughing to citric acid was most significant for camphor and also significant for thymol nasal challenge, again, data for 1,8 -cineole did not reach the level of statistical significance $(17 \pm 3$ vs $13 \pm 3$ vs $7 \pm 2$, p<0.05) in order 1,8-cineole-thymol-camphor. ${ }^{*} \mathrm{p}<0.05$, med - median, IQR - interquartil range, OVA - ovalbumin. 
strated the effect of pathological process in the upper airways on coughing. Observed reduction of coughing to citric acid was most significant for camphor and also significant for thymol nasal challenge. Data for 1,8 -cineole did not reach statistical significance (17 \pm 3 vs. $13 \pm 3$ vs. $7 \pm 2, \mathrm{p}<0.05$ ) in order 1,8 -cineole-thymol-camphor. (Fig. 3).

\section{DISCUSSION}

This study demonstrated that intranasal administration of thymol and camphor $(0.015 \mathrm{ml}$, $10^{-3} \mathrm{M}$ each) significantly reduced total count of coughs and prolonged the cough latency in the conscious guinea pig model of allergic upper airway inflammation. 1,8-cineole only showed the tendency to reduce coughing, however, the data did not reach statistical significance.

At the other hand no one of selected aromatic substances influenced the onset, duration or the magnitude of the nasal symptoms induced by the nasal administration of the allergen ovalbumin. In our experiment, trained observers were in charge to measure and quantify the magnitude and duration of nasal symptoms provoked by nasal allergen challenge as the only way of objective assessment. Although this method was validated to objectively measure and quantify nasal symptom score, we failed to observe any changes. This finding is in agreement with previously published data that aromatic substances mainly improve the subjective interpretation and perception of reduced upper airway symptoms, however, the objective measurement - for example acoustic rhino-manometry remain unchanged $[20,21]$. And since the animal studies lack the subjective aspect, we have to conclude, that effect of thymol, camphor and 1,8-cineole pre - treatment on nasal symptoms in allergic rhinitis is not considerable.

Nasal administration of these substances, except of the 1,8-cineole, significantly prolonged the cough latency and reduced the total count of coughs induced by citric acid. The data from study performed on naive animals were obtained after inhalation of vaporized camphor, thymol and 1,8-cineole [6]. Although the guinea pigs are obligatory nasal breathers, it is probable that considerable amount of the vapours was trapped in the nasal passages and that only a small proportion reached the lower airways. Therefore the effect of nasal sensory afferents in cough down- regulation cannot be excluded in that study [6].

It is known, that terpenes have also direct effect on vessels, smooth muscles and glands in the airways [22] and these direct effects must be taken into consideration when possible antitussive action is discussed. However, our experiment was designed to apply the substances exclusively to the upper airway in $0.015 \mathrm{ml}$ volume. It was identified using the Evans blue dye that this volume is distributed over the nasal mucosa, and no staining was observed in the more distal airways. Since the airways were not separated, it is not completely possible to exclude the alternative that some of the molecules of terpenes were inhaled to the lower airways, but we suppose, that major effects were induced in the nasal mucosa.

The antitussive affect of our tested substances after their administration to the nose together with the data obtained in our previous studies $[8,9]$ indicate that antitussive effect of aromatic substances is probably mediated by trigeminal sensory afferents, possibly involving olfactory and supramedullar influences.

While the study in naive animals documented that 1,8-cineole has the most potent antitussive effect [6] our data indicate that 1,8-cineole provided weak, statistically not significant effect on cough latency and total cough count. This conflicting evidence could be a consequence of the nature and the design of the experiments (naive versus allergic animals, inhalation of vaporized cineole versus intranasal administration of it) and finally natural variability of the studied populations. A confounding feature of all studies where multiple challenges are performed on animals is a variation in response due to high variability of the conscious guinea pigs cough response. This factor could be responsible for non significance 
in final statistical evaluation. Another factor must be taken into consideration. It is the amount of the cineole and also all of the substances applied to the nose with ability to stimulate nasal afferents as the guinea pigs may expel some amount of it as soon as it was applied. It is possible to anaesthetize the nasal mucosa prior to the challenge of allergen for example, but this modification is absolutely in disagreement with the concept when the role of the nasal afferents is investigated [23].

It is known that antitussive effect of menthol - one of the aromatic terpenes is exclusively mediated by TRPM8 expressing afferents, which produce different sensations when comparing to TRPV1. TRPV1 activation is known to either provoke or facilitate coughing $[15,16]$. In our experiment we used TRPM8 agonists - camphor and 1,8-cineole, but also thymol. Thymol and camphor are known TRPV3 agonists [24] and their intranasal administration reduced coughing as well. This is indirect evidence, that cough suppressing effect is not necessarily channel - specific process. The results obtained after the camphor and thymol challenges could be circumstantial evidence about the importance of TRPV1 as the channel responsible for induction and up-regulation of cough, as they both strongly desensitize TRPV1 ion channel [14]. Camphor also activates and sensitizes TRPM8 channel, which is in agreement with our previous findings [8,9].

It is speculated that mechanism of the action for all aromatic substances applied to the nose (menthol, thymol, cineole, camphor) is mediated via changes of the breathing pattern. We did not measure the respiratory rate and the tidal volume through the entire experiment, because it is modified by citric acid inhalation which considerably influences the breathing pattern and the airway lumen [24, 25]. There are studies in which the authors described reduction of ventilation followed by reduction of the cough response after nasal challenges of water, allyl-iso-thiocyanate and menthol respectively [26, 27].

It is generally accepted, that respiratory and - cough related neuronal networks overlap significantly and that stimuli that increase ventilatory drive also frequently potentiate cough and vice versa, the depression of ventilation usually results in the depression of cough [28, 29] at least in rodents.

The explanation relies so far on a mechanistic understanding of the airway reflexes, where cough with its deep inspiration should be inhibited to prevent "aspiration" of the substances entering the nose [27]. Whether this orchestration of the airway protective and defensive reflexes exists is not know at the moment and further studies are necessary to clarify interaction between individual airway reflex responses.

Cough is modulated by the cortical influences [30] and many other factors like behavioural, attentional, cognitive influences [31]. In this aspect it is very important to note olfactory signalling, or trigeminal-olfactory relationships. The nature and extend of interactions between trigeminal and olfactory stimulation are poorly understood however TRPM8 and TRPV3 were detected both on trigeminal and olfactory nerve terminals [32]. For example exposure of the nose to the malodorants increases the responsiveness of trigeminal nerves, and this mechanism is mediated by paracrine signalling pathway between olfactory and trigeminal nerves [33]. Also, patients with acquired olfactory loss exhibit reduced trigeminal sensitivity, possibly due to the lack of the interactions. Therefore the contribution of the olfactory influences must be considered.

In conclusion, we demonstrated that nasal administration of the camphor and thymol significantly reduces coughing induced by citric acid in the animal model of airway hyperresponsiveness. Empirical use of the over-the-counter medication against cough and common cold is getting stepwise explained by our recent understanding of the cough plasticity, ion channels activation, and the role of some natural ligands capable to activate them (34). Further studies are necessary to clarify for example the effects of aromatherapy and other approaches using natural volatile substances. The message from our study for symptomatic treatment of the airway diseases is very promising, as the most frequently used preparation as nasal drops, sprays or instillations. As it was demonstrated, even intranasal administration of aromatic substances can suppress coughing via mechanisms of cough plasticity. 


\section{REFERENCES}

1. Kreindler JL, Chen B, Kreitman Y, Kofonow J, Adams K, Cohen NA. The novel dry extract BNO 1011 stimulates chloride transport and ciliary beat frequency in human respiratory epithelial cultures. Am J Rhinol and Allergy 2012; 26 (6): 439-443.

2. Kemmerich B, Eberhardt B, Stammer H. Efficacy and tolerability of a fluid extract combination of the thyme herb and ivy leaves and matched placebo in adults suffering from acute bronchitis with productive cough. Arzneim-Forsch/Drug Research 2006; 56 (9): 652-660.

3. Jund R, Mondilger M, Steindl H, Stammer H, Stierna P, Bachert C. Clinical efficacy of a dry exracts of five herbal drugs in acute viral rhinosinusitis. Rhinology 2012; 50: 417-426.

4. Glatthaar-Saalmuller B, Rauchhaus U, Rode S, Haunschild J, Saalmuller A. Antiviral activity in vitro of two preparation of the herbal medicinal product Sinupret against viruses causing respiratory infection. Phytomedicine 2011; 19: 1-7.

5. Rossi A, Dehm F, Kiesselbach Ch, Haunschild J. The novel Sinupret dry extract exhibits anti-inflammatory effectiveness in vivo. Fitoterapia 2012; 83: 715-720.

6. Laude EA, Morice AH, Grattan TJ. The antitussive effects of menthol, camphor and cineole in conscious GP. Pulm Pharmacol 1994; 7 (3): 179-184.

7. Gavliakova S, Biringerova Z, Buday T, Brozmanova M, Calkovsky V, Poliacek I, Plevkova J. Antitussive effects of nasal thymol challenges in healthy volunteers. Respir Physiol Neurobiol. 2013; 187(1):104-7.

8. Plevkova J, Kollarik M, Poliacek I, Brozmanova M, Surdenikova L, Tatar M, Mori N, Canning BJ. The role of trigeminal nasal TRPM8-expressing afferent neurons in the antitussive effects of menthol. J Appl Physiol 2013; 115(2): 268-74.

9. Buday T, Brozmanova M, Biringerova Z, Gavliakova S, Poliacek I, Calkovsky V, Shetthalli MV, Plevkova J. Modulation of cough response by sensory inputs from the nose - role of trigeminal TRPA1 versus TRPM8 channels. Cough 2012;3(1):11 -18

10. McKemy DD. How cold is it? TRPM8 and TRPA1 in molecular logic of cold sensation. Mol Pain 2005; 1:16.doi: $10.1186 / 1744-8069$

11. Kobayashi K, Fukuoka T, Obata K, Yamanaka H, Dai Y, Tokunaga A, Noguchi K. Distinct expression of TRPM8, TRPA1 a TRPV1 $\mathrm{m}$ RNA in rat primary afferent neuron with Ad/C fibers and colocalization with trk receptors. J Comp Neurol 2005; 493: 596-606.

12. Abe J, Hosakawa H, Okazawa M. TRPM8 protein localisation in trigeminal ganglia and taste papillae. Molecular Brain Research 2005; 136 (2) 91-98.

13. Xu H, Ramsey IS, Kotecha SA, Moran MM, Chong JA, Lawson D, Ge P, Lilly J, Silos-Santiago I, Xie Y, DiStefano PS, Curtis R, Clapham DE. TRPV3 is a calcium-permeable temperature-sensitive cation channel. Nature 2002; 418(6894): 181-186.

14. Xu H, Blair NT, Clapham DE. Camphor activates and strongly desensitizes the transient receptor potential vanilloid subtype 1 channel in a vanilloid-independent mechanism. J Neurosci 2005, 25(39):8924-8937.

15. Morice AH, Geppetti P. Cough. 5: The type 1 vanilloid receptor: a sensory receptor for cough. Thorax 2004; 59(3):257-258.

16. Plevkova J, Kollarik M, Brozmanova M, Revallo M, Varechova S, Tatar M. Modulation of experimentally-induced cough by stimulation of nasal mucosa in cats and guinea pigs Respir Physiol Neurobiol 2004; 142: 225-235.

17. Canning BJ, Mori N. Encoding of the cough reflex in anaesthetized guinea pigs. Am J Physiol Regul Integr Comp Physiol 2011; 300(2):369-377.

18. Brozmanova M, Plevkova J, Tatar M, Kollarik M. Cough reflex sensitivity is increased in the guinea pig model of allergic rhinitis. Journal of physiology and pharmacology 2008; 59 (6):153-161.

19. Underwood S, Foster M, Raeburn D, Bottoms S, Karlsson JA. Time-course of antigen-induced airway inflammation in the guinea-pig and its relationship to airway hyperresponsiveness. Eur Respir J 1995; 8(12):2104-13.

20. Galeotti N, Di Cesare Mannelli L, Mazzanti G, Bartolini A, Ghelardini C. Menthol: a natural analgesic compound. Neurosci Lett 2002; 322(3):145-8.

21. R. Eccles. Menthol: effects on nasal sensation of airflow and the drive to breathe. Curr Allergy Asthma Rep 2003; 3: 210-214

22. Millqvist E, Ternesten-Hasséus E, Bende M. Inhalation of menthol reduces capsaicin cough sensitivity and influences inspiratory flows in chronic cough. Respiratory Medicine 2013; 107 (3): 433-438

23. T Nabe, N. Mizutani, K. Shimizu, H. Takenaka, S. Kohno. Development of pollen-induced allergic rhinitis with early and late phase nasal blockage in guinea pigs Inflammation Research 1998; 47 (9): 369 -374

24. Tatar M, Sant'Ambrogio G, Sant'Ambrogio F B. Laryngeal and tracheobronchial cough in anesthetized dogs. J Appl Physiol 1994; 76 (6): 2672-9.

25. Ricciardolo FL, Rado V, Fabbri LM, Sterk PJ, DiMaria GU, Geppetti P. Bronchoconstriction induced by citric acid inhalation in guinea pigs: role of tachykinins, bradykinin, and nitric oxide. Am J Respir Crit Care Med 1999; 159 (2): 557-62.

26. Biringerova Z, Gavliakova S, Brozmanova M, Tatar M, Hanuskova E, Poliacek I, Plevkova J. The effects of nasal irritant induced responses on breathing and cough in anaesthetized and conscious animal models. Respir Physiol Neurobiol 2013; doi:pii: S1569-9048(13)00274-7. 10.1016/j.resp.2013.08.003. 
27. Poussel M, Varechova S, Demoulin B, Chalon B, Schweitzer C, Marchal F, Chenuel B Nasal stimulation by water down-regulates cough in anesthetized rabbits. Respir Physiol Neurobiol 2012; 183(1):20-25.

28. Tatar M, Nagyova B. Abdominal capsaicin-sensitive receptor activation and mechanically induced cough in anesthetized cats. International Union of Physiological Science, Prague 1991 (98).

29. Tatar M, Nagyova B, Widdicombe JG. Veratrine-induced reflexes and cough. Respir Med 1991; 85(A): 51-55.

30. Widdicombe J, Eccles R, Fontana G. Supramedullary influences on cough. Respir Physiol Neurobiol 2006, 52(3):320-328.

31. Van den Bergh O, Van Diest I, Dupont L, Davenport PW. On the Psychology of Cough. Lung 2011.

32. Keh SM, Facer P, Yehia A, Sandhu G, Saleh HA, Anand P. The menthol and cold sensation receptor TRPM8 in normal human nasal mucosa and rhinitis. Rhinology. 2011; 49(4):453-7.

33. Desesa CR, Vaughan RP, Lanosa MJ, Fontaine KG, Morris JB. Sulfur-containing malodorant vapours enhance responsiveness to the sensory irritant capsaicin. Toxicol Sci 2008;104(1):198-209.

34. Jurecek L, Nosalova G, Hromadkova Z, Kostalova Z. Antitussive activity of extracts from Fallopia Sachalinenesis. Acta Medica Martiniana 2012, Suppl. 1, 24-30.

\section{List of abbreviations}

CA - citric acid

OVA - ovalbumin

TRPM8 - transient receptor potential channel, melastin subgroup member 8

TRPV3 - transient receptor potential channel, vanilloid subgroup member 3

TRPV1- transient receptor potential channel, vanilloid subgroup member 1

TRPV1+ - neurons expressing TRPV1 channel

sc-rt-PCR - single cell reverse transcription and polymerase chain reaction

\section{Acknowledgement}

The study was supported by VEGA no 1/0031/11 and Center of Experimental and Clinical Respirology II (CEKR) co financed from EU sources.

Received: September, 5, 2013

Accepted: October 21, 2013 